\title{
Nonstandard Finite Difference Scheme for a Computer Virus Model
}

\author{
Mehmet KOCABIYIK 1, Nihal ÖZDOĞAN ${ }^{2 *}$ Mevlüde Yakıt ONGUN \\ ${ }^{1}$ Department of Mathematics, Burdur Mehmet Akif Ersoy University, Burdur, Turkey \\ 2* Department of Mathematics, Bursa Technical University, Bursa, Turkey \\ ${ }^{3}$ Department of Mathematics, Süleyman Demirel University, Isparta, Turkey.
}

Cite this paper as:

Kocabiyik, M., Özdoğan, N., Ongun, Y,M. (2020). Nonstandard Finite Difference Scheme for a Computer Virus Model. Journal of Innovative Science and Engineering. 4(2): 96-108

*Corresponding author: Nihal ÖZDOĞAN E-mail: nihal.ozdogan@btu.edu.tr Tel: +90 (224) 3003848

Received Date: $18 / 03 / 2020$

Accepted Date: 07/10/2020

(C) Copyright 2020 by

Bursa Technical University. Available online at http://jise.btu.edu.tr/

\section{(c) (1) (9)}

The works published in Journal of Innovative Science and Engineering (JISE) are licensed under a Creative Commons Attribution-NonCommercial 4.0 International License.

\begin{abstract}
This study introduces us to a new model developed for computer viruses. The model is presented to remove the protective restriction on the total number of computers connected to the Internet. This model is nonlinear differential equation system. Therefore, finding analytical solutions is very difficult. This means that we have to apply numerical methods in order to find the solution. The behavior of numerical solution has been investigated for the discretized system. By using Nonstandard Finite Difference Scheme (NSFD), it is aimed to preserve both the positivity of the solutions for positive initial points and the local asymptotic stability of the equilibrium point.
\end{abstract}

Keywords: Computer virus, Nonstandard Finite Difference Scheme, Discretization, Internet. 


\section{Introduction}

A computer virus is a program that can infect other programs by changing them to include an evolved copy of its own. When a computer has a virus attack, the virus can perform destructive operations such as changing data, deleting data, deleting files, codifying files and formatting disks [1,2].

The computer virus began to attract attention in the world in the 1980s and it has been becoming a big threat in our daily life. In recent years, this threat has been even more serious with the improvement of hardware and software technology and the increasing importance of computer networks. For example, the personal data from network users such as bank accounts and passwords are likely to cause serious harm to persons and corporations. Therefore, it is inevitable to suggest various mathematical models to investigate the control and diffusion of computer virus and to define their behaviour. An anti-virus software program is the most important way to defend against viruses in these days. Nevertheless, the improvement of new anti-virus software program is always behind the appearance of new viruses; therefore, it can not help to bring out long-range policies that contain viruses. That is the reason why the solutions of virus programs with mathematical models have become more and more important [3-8].

Many research on the computer viruses, such as creating of new viruses and new vectors or the development of new techniques for invention and inclusion, has been conducted to explore how computer viruses work. However, recent studies were limited basically to three epidemic models: the SI model $[9,10]$, the SIS model [10-20] and the SIR model $[13,21-24]$.

Making the formulation of the model for shortness computers are called as nodules. A nodule is called "internal" or "external", depending on whether it is connected to the Internet. A nodule is defined as infected or uninfected, depending on whether it contains viruses. An infected nodule is known as latent if the viruses in it are all latent. An infected nodule is called as breaking-out if at least one virus in it is breaking-out. Based on the former studies, all internal nodules are classified into uninfected internal nodules ( $\mathrm{S}$ nodules), hidden internal nodules (L nodules), and fragmented internal nodules (B nodules). Moreover, all external nodules are classified into uninfected external nodules ( $S^{*}$ nodules), hidden external nodules ( $L^{*}$ nodules) and broken external nodules ( $B^{*}$ nodules) [4,5]. Let $S(t), L(t)$ and $B(t)$ indicate the points of $S, L$ and $B$ nodules at time $t$ respectively. The model on which we are working depends on the following acceptances:

(A1) If $\mu_{1}>0, S^{*}$ nodules are bonded to the Internet at the fixed rate.

If $\mu_{2}>0, L^{*}$ nodules are are bonded to the Internet at the fixed rate. Let $=\mu=\mu_{1}+\mu_{2}$.

(A2) $\delta>0$ is a constant probability for every internal nodules are disconnected from the Internet.

(A3) $\theta>0$ is a constant probability for every $\mathrm{S}$ nodules are infected.

(A4) For $\beta_{1}>0, \beta_{2}>0$,

$\beta_{1} L(t)$ : The probability function for every infected $S$ nodules by L nodules,

$\beta_{2} B(t)$ : The probability function for every infected $S$ nodules by B nodules.

(A5) $\alpha>0$ is a constant probability for each break out $L$ nodules.

(A6) $\omega_{1}>0$ is a constant probability for each cured $L$ nodules and 
$\omega_{2}>0$ is a constant probability for each cured $B$ nodules.

Based on these acceptances, the investigated computer virus model is formulated as [5];

$$
\begin{aligned}
\frac{d S}{d t} & =\mu_{1}-\left(\beta_{1} L+\beta_{2} B\right) S+\omega_{1} L+\omega_{2} B-(\delta+\theta) S, \\
\frac{d L}{d t} & =\mu_{2}+\left(\beta_{1} L+\beta_{2} B\right) S-\left(\omega_{1}+\alpha+\delta\right) L+\theta S, \\
\frac{d B}{d t} & =\alpha L-\left(\omega_{2}+\delta\right) B .
\end{aligned}
$$

$(S(0), B(0), L(0))$ are real numbers and initial conditions.

In this study, the stability analysis of the Equations (1) - (3) will be presented with Nonstandard Finite Difference Schemes (NSFD). As the most important feature of this method, a suitable function that depends on $h$ can be selected instead of the step size $h$. This scheme allows to find better results for the nonlinear model. The procedures for the NSFD method were introduced by Mickens in 1989 [25].

Using the appropriate denominator function prevents the inconsistency of the solutions of the model. To see how to select the denominator function and how to implement it to different models, you can look at [25-32] .

The format of this paper is arranged as follows: Section 2 gives some definitions and theorems about NSFD, which is the discretization method to be used in solving linear and nonlinear equations. Section 3 reports the discretization of the computer virus model. Section 4 first includes the useful information about the stability analysis, and then presents the findings related to the stability analysis of the system. Section 5 comprises some graphics and the simulations of the model with different parameters. Section 6, which is the last section, contains some discussions and conclusions.

\section{Nonstandard Finite Difference Schemes for ODEs}

Micken's NSFD method can be expressed as follows using [27-33]. Consider the following ordinary differential equation:

$$
\frac{d x}{d t}=f(x, \lambda)
$$

Here $\lambda$ is a parameter. Basic nonstandard finite difference schemes are

$$
t \rightarrow t_{n}=h n, \quad x(t) \rightarrow x_{n}, f(x) \rightarrow f\left(x_{n}\right), \quad \frac{d x}{d t} \rightarrow \frac{x_{n+1}-x_{n}}{\phi(h)} .
$$

Here $\phi(h)$ depends on the step size of $\Delta t=h$ and satisfies $\phi(h)=h+O\left(h^{2}\right)$. Therefore, the denominator function $\phi(h)$, should be chosen as, 


$$
\phi(h)=\frac{1-e^{(-K h)}}{K}
$$

where $\mathrm{K}$ is calculated from the information of the fixed points of (4). Here $\phi(h)$ is an increasing function of $h$ and it may hinge on the parameters appearing in the differential equations. The discretization of nonlinear terms using NSFD can be done as following examples ;

$$
\begin{aligned}
& x^{2} \rightarrow x_{n+1} x_{n}, \\
& x^{2} \rightarrow \frac{x_{n+1}+x_{n}+x_{n-1}}{3} x_{n}, \\
& x^{3} \rightarrow \frac{x_{n+1}+x_{n-1}}{2} x_{n}{ }^{2} .
\end{aligned}
$$

The decay equation is an example for NFSD discretization process and its application.

$$
\frac{d x}{d t}=-\alpha x
$$

here $\alpha$ is a constant. The discretization scheme is [31],

$$
\frac{x_{n+1}-x_{n}}{\phi}=-\alpha x_{n}, \quad \phi(h, \alpha)=\frac{1-e^{-\alpha h}}{\alpha} .
$$

In another basic example,

$$
\frac{d x}{d t}=\alpha_{1} x-\alpha_{2} x^{2}
$$

here the NSFD scheme is given as follows [31],

$$
\frac{x_{n+1}-x_{n}}{\phi}=-\alpha_{1} x_{n}-\alpha_{2} x_{n+1} x_{n}, \phi\left(h, \alpha_{1}\right)=\frac{e^{\alpha_{1} h}-1}{\alpha_{1}} .
$$




\section{Discretization of the Computer Virus Model}

Let $S(t)+L(t)+B(t)=N(t)$. By adding and simplifying the triple system (1) - (3) side by side, we get the following equation:

$$
\frac{d N(t)}{d t}=\mu-\delta N(t)
$$

implying $\lim _{t \rightarrow \infty} N(t)=\frac{\mu}{\delta}$. This shows that the total number of internal computers approaches the constant $N^{*}=\frac{\mu}{\delta}$.

The above equation is a linear nonhomogeneous ordinary differential equation with constant coefficient. To solve Equation (11) with Nonstandard Finite Difference Scheme, the following discretization will be done:

$$
\frac{d N}{d t} \rightarrow \frac{N_{i+1}-N_{i}}{\phi_{1}} \text { and } N \rightarrow N_{i+1}
$$

Using the discretization in Equation (12), Equation (11) is written as follows:

$$
N_{i+1}=\frac{N_{i}+\phi_{1} \mu}{1+\delta \phi_{1}}
$$

Instead of $S, N-L-B$ is written in the systems (2)-(3) and the following discretizations (14)-(15) are done by NSFD,

$$
\begin{gathered}
\frac{d L}{d t} \rightarrow \frac{L_{i+1}-L_{i}}{\phi_{2}} \\
\frac{d B}{d t} \rightarrow \frac{B_{i+1}-B_{i}}{\phi_{3}} .
\end{gathered}
$$

And the equation system (16) can be written:

$$
\begin{aligned}
& N_{i+1}=\frac{N_{i}+\phi_{1} \mu}{1+\delta \phi_{1}} \\
& L_{i+1}=\frac{L_{i}+\phi_{2}\left(\mu_{2}+\left(N_{i}-B_{i}\right)\left(\beta_{1} L_{i}+\beta_{2} B_{i}+\theta\right)\right)}{1+\phi_{2}\left(\beta_{1} L_{i}+\beta_{2} B_{i}+\theta+\omega_{1}+\alpha+\delta\right)} \\
& B_{i+1}=\frac{B_{i}+\phi_{3} \alpha L_{i}}{1+\phi_{3}\left(\omega_{2}+\delta\right)} .
\end{aligned}
$$

The denominator functions of Equation (16) are chosen as, 


$$
\begin{aligned}
& \phi_{1}=\frac{e^{\delta h}-1}{\delta}, \\
& \phi_{2}=\frac{e^{\left(\theta+\omega_{1}+\alpha+\delta\right) h}-1}{\theta+\omega_{1}+\alpha+\delta}, \\
& \phi_{3}=\frac{e^{\left(\omega_{2}+\delta\right) h}}{\omega_{2}+\delta} .
\end{aligned}
$$

\section{Stability Analysis}

This section gives some useful information about the local asymptotic stability of discrete systems. In particular, the following information is important to examine the Schur-Cohn criterion, which deals with the coefficient matrix of the linearized system:

$1-\operatorname{trJ}+\operatorname{det} J>0$

$1+\operatorname{trJ}+\operatorname{det} J>0$

$\operatorname{det} J>1$

where, $\mathrm{J}$ and trJ denote the coefficient matrix and the trace of the matrix of the linearized systems, respectively. These are some suggestions about Schur-Cohn criteria and its use [29, 34-42].

Lemma 1. (Schur-Cohn Criteria) For the quadratic characteristic equation $P(\lambda)=\lambda^{2}+p_{1} \lambda+p_{2}$ the roots satisfy $\left|\lambda_{k}\right| \leq$ $1, k=1,2$ but if the following conditions are satisfied [36]:

$P(1)=1+p_{1}+p_{2}>0$

$P(-1)=1-p_{1}+p_{2}>0$

$p_{2}<1$

Lemma 2. (Jury Condition) Suppose the characteristic polynomial $P(\lambda)=\lambda^{3}+p_{1} \lambda^{2}+p_{2} \lambda+p_{3}$ The solutions $\lambda_{k}, k=$ $1,2,3$ of $P(\lambda)=0$ satisfy $\left|\lambda_{k}\right| \leq 1$ if and only if the next three conditions are applied [36]:

$P(1)=1+p_{1}+p_{2}+p_{3}>0$, 
$(-1)^{3} P(-1)=1-p_{1}+p_{2}-p_{3}>0$,

$1-\left(p_{3}\right)^{2}>\left|p_{2}-p_{1} p_{3}\right|$

Theorem 1. The discretized system (16) has only one fixed point:

$E^{*}=\left(N^{*}, L^{*}, B^{*}\right)=\left(\frac{\mu}{\delta}, \frac{-k_{2}+\sqrt{\left(k_{2}\right)^{2}-4 k_{1} k_{3}}}{2 k_{1}}, \frac{\alpha L}{\omega_{2}+\delta}\right)$

here,

$k_{1}=-\beta_{1}$

$k_{2}=\beta_{2} B+\theta+\omega_{1}+\alpha+\delta-N \beta_{1}+B \beta_{1}$

Proof. Since we write $N^{*}$ instead of $N_{i+1}$ and $N_{i}$ in the first equation of system (16), the following equation can be found

$$
N^{*}=\frac{N^{*}+\phi_{1} \mu}{1+\delta \phi_{1}}
$$

With the required multiplication

$$
N^{*}\left(1+\delta \phi_{1}\right)=N^{*}+\phi_{1} \mu \rightarrow N^{*}+N^{*} \delta \phi_{1}=N^{*}+\phi_{1} \mu \rightarrow N^{*} \delta \phi_{1}=\phi_{1} \mu,
$$

the expression $N^{*}$ is obtained as $\frac{\mu}{\delta}$.

In the second equation of system (16) if we write $L^{*}$ instead of $L_{i+1}$ and $L_{i}$ with the same method, the following equation can be found:

$$
L^{*}=\frac{L^{*}+\phi_{2}\left(\mu_{2}+\left(N_{i}-B_{i}\right)\left(\beta_{1} L^{*}+\beta_{2} B_{i}+\theta\right)\right)}{1+\phi_{2}\left(\beta_{1} L^{*}+\beta_{2} B_{i}+\theta+\omega_{1}+\alpha+\delta\right)} .
$$

If the equation (19) is rearranged with the help of system (18), Equation (19) can be written as

$$
\begin{aligned}
& k_{1}\left(L^{*}\right)^{2}+k_{2} L^{*}+k_{3}=0, \\
& L^{*}=\frac{-k_{2}+\sqrt{\left(k_{2}\right)^{2}-4 k_{1} k_{3}}}{2 k_{1}} .
\end{aligned}
$$

If the same operations are done respectively for $B_{i+1}$ the following equation can be found: 


$$
B^{*}=\frac{\alpha L}{\omega_{2}+\delta}
$$

Theorem 2. The fixed point $E^{*}$ is local asymptotic stable.

Proof. The Jacobian matrix of system (16) has given by the form $J=\left(c_{i j}\right)_{3 \times 3}$,

$$
\left[\begin{array}{ccc}
\frac{1}{1+\delta \phi_{1}} & 0 & 0 \\
c_{21} & c_{22} & c_{23} \\
0 & c_{32} & c_{33}
\end{array}\right]
$$

here,

$$
\begin{aligned}
& c_{21}=\frac{\phi_{2}\left(B \beta_{2}+L \beta_{1}+\theta\right)}{1+\phi_{2}\left(B \beta_{2}+L \beta_{1}+\theta+\alpha+\omega_{1}+\delta\right)} \\
& C_{22}=\frac{1+\phi_{2}(N-B) \beta_{1}}{1+\phi_{2}\left(B \beta_{2}+L \beta_{1}+\theta+\alpha+\omega_{1}+\delta\right)}-\frac{\left(L+\phi_{2}\left(\mu_{2}+(N-B)\left(B \beta_{2}+L \beta_{1}+\theta\right)\right)\right) \phi_{2} \beta_{1}}{\left(\left(1+\phi_{2}\left(B \beta_{2}+L \beta_{1}+\theta+\alpha+\omega_{1}+\delta\right)\right)\right)^{2}} \\
& c_{23}=\frac{\phi_{2}\left(-B \beta_{2}-L \beta_{1}-\theta+(N-B) \beta_{2}\right)}{1+\phi_{2}\left(B \beta_{2}+L \beta_{1}+\theta+\alpha+\omega_{1}+\delta+\omega_{1}\right)}-\frac{\left(L+\phi_{2}\left(\mu_{2}+(N-B)\left(B \beta_{2}+L \beta_{1}+\theta\right)\right)\right) \phi_{2} \beta_{1}}{\left(\left(1+\phi_{2}\left(B \beta_{2}+L \beta_{1}+\theta+\alpha+\omega_{1}+\delta\right)\right)\right)^{2}} \\
& C_{32}=\frac{\phi_{3} \alpha}{1+\phi_{3}\left(\omega_{2}+\delta\right)} \\
& c_{33}=\frac{1}{1+\phi_{3}\left(\omega_{2}+\delta\right)^{\circ}}
\end{aligned}
$$

The characteristic equation of Jacobian matrix can be found:

$$
\left(\lambda-\frac{1}{1+\delta \phi_{1}}\right)\left(\lambda^{2}+t_{1} \lambda+t_{2}\right)=0, \quad J=\left[\begin{array}{ll}
c_{22} & c_{23} \\
c_{32} & c_{33}
\end{array}\right]
$$

It is sufficient to use the trace and det expressions for $t_{1}$ and $t_{2}$. In this case $t_{1}$ and $t_{2}$ can be written as

$$
t_{1}=- \text { Trace } J=-\left(c_{22}+c_{23}\right), \quad t_{2}=\text { Det } J=\left(c_{22} \cdot c_{33}-c_{23} \cdot c_{32}\right) .
$$


The first eigenvalue is $\lambda_{1}=\frac{1}{1+\delta \phi_{1}}$ and the other eigenvalues can be found as $\left(\lambda^{2}+t_{1} \lambda+t_{2}\right)$ solving this equation. Also, the following conditions are satisfied for local asymptotic stability of the fixed point $E^{*}$ :
i) $1+t_{1}+t_{2}>0$,
ii) $1-t_{1}+t_{2}>0$

iii) $t_{2}<1$

\section{Simulations}

In this section, we consider the model (1)-(3) with different parameters. In Figure 1, the numerical solutions are obtained by NSFD method with different initial conditions and with the parameters:

$$
\beta_{1}=0.01 \beta_{2}=0.08 \omega_{1}=0.2 \omega_{2}=0.1 \alpha=0.2 \delta=0.1 \quad \theta=0.2 \mu_{1}=4 \mu_{2}=2 .
$$

Equilibrium point is obtained as $(7.73,26.13,26.13)$.
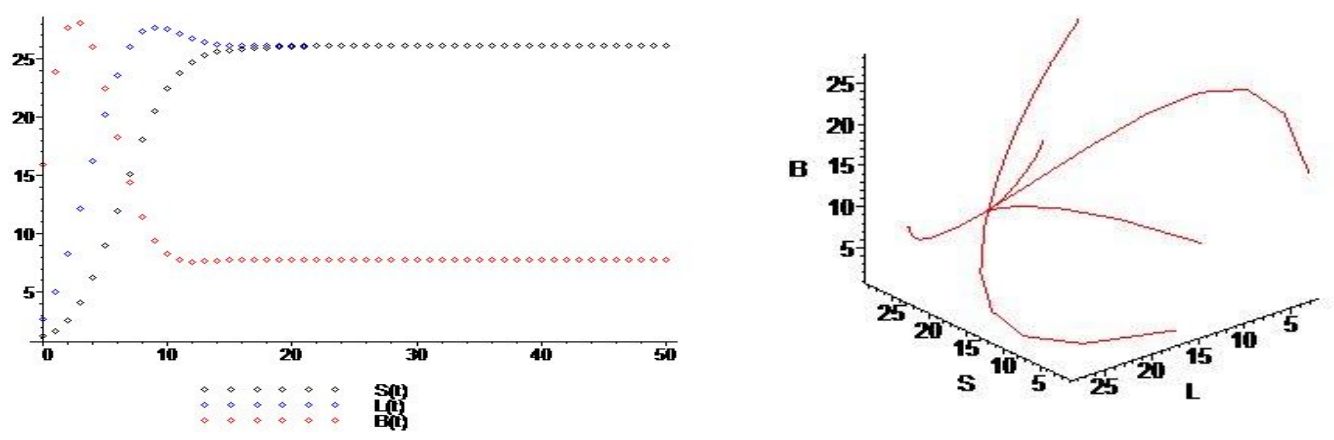

Figure 1. Equilibrium point $=(7.73,26.13,26.13)$.

In Figure 2, we simulate the solutions and the phase portraits with the parameters:

$$
\beta_{1}=0.05 \beta_{2}=0.08 \omega_{1}=0.2 \omega_{2}=0.1 \alpha=0.1 \delta=0.2 \quad \theta=0.1 \mu_{1}=5 \mu_{2}=2 \text {, }
$$

and equilibrium point is obtained as $(5.42,18.42,6.14)$. 

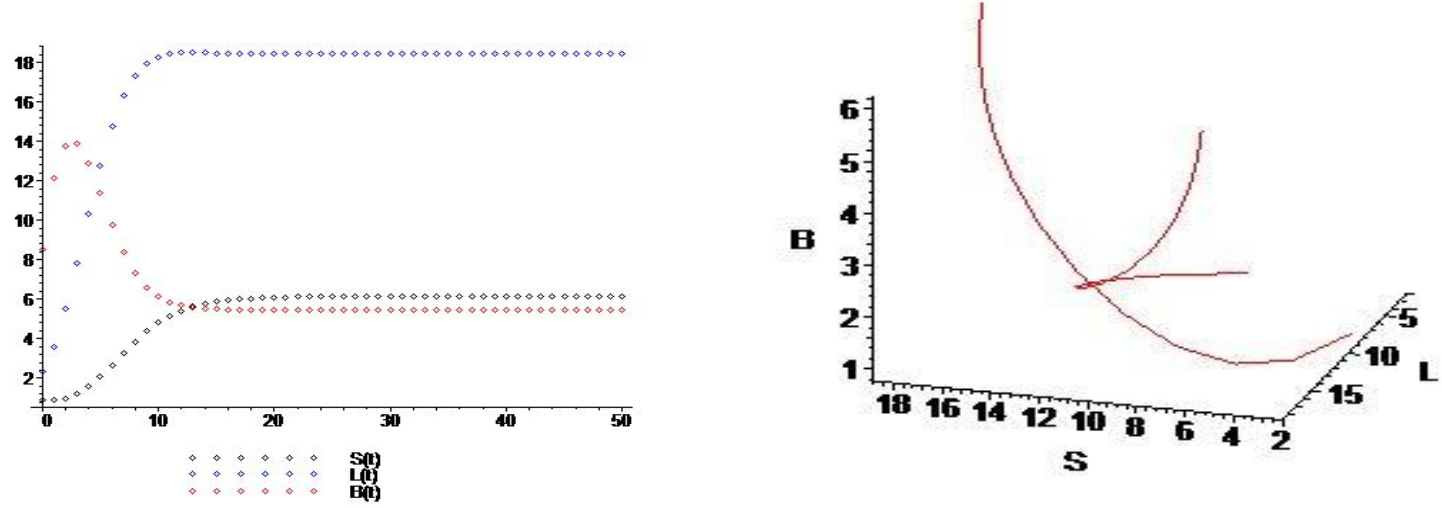

Figure 2. Equilibrium point $=(5.42,18.42,6.14)$.

In Figure 3, we consider the model with the parameters:

$$
\beta_{1}=0.04 \beta_{2}=0.05 \omega_{1}=0.2 \omega_{2}=0.1 \alpha=0.2 \delta=0.1 \quad \theta=0.1 \mu_{1}=1.5 \mu_{2}=1 \text {. }
$$

Equilibrium point is obtained as $(4.06,10.46,10.46)$.
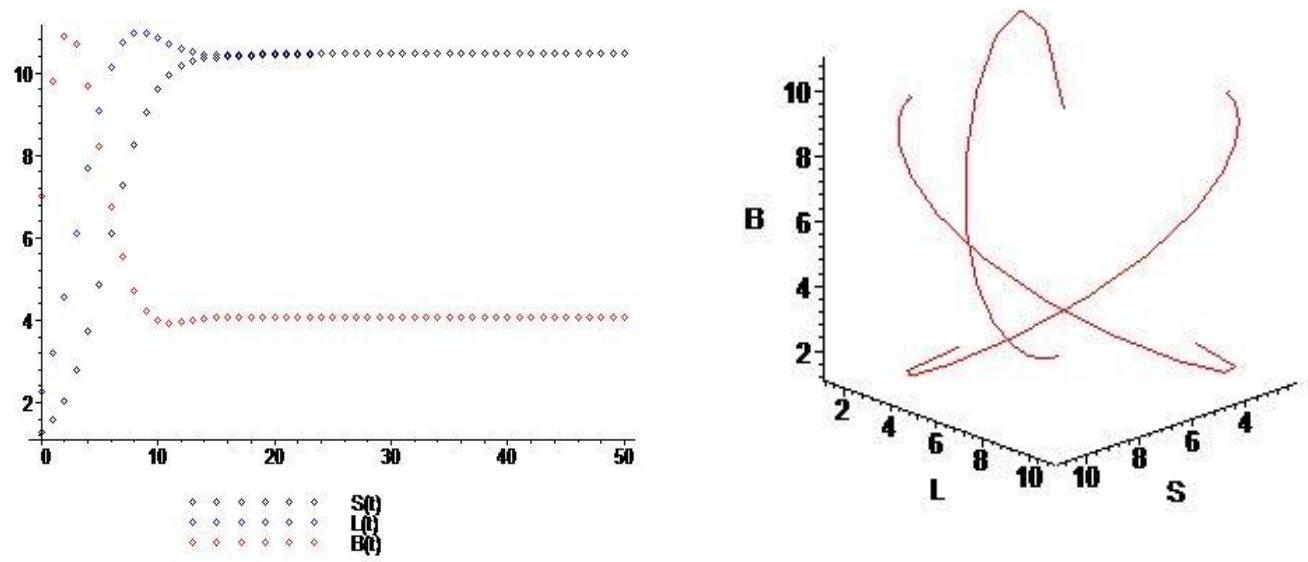

Figure 3. Equilibrium point $=(4.06,10.46,10.46)$.

This graphics and simulations are appropriate with $[4,5]$. 


\section{Conclusion}

In this paper, firstly, computer virus model given by Equation (1)-(3) was discretized using NSFD method. Instead of $S$, $N-L-B$ was written in the Equation (2) and Equation (3). Then, stability analysis was done with the help of some lemmas and theorems for the discretized system given by Equation (16). Some graphics and simulations of the solutions with different parameters were given. Using these solutions, the current study have made significant contribution to the relevant research by providing important insights into the spread of computer viruses on the Internet.

\section{Acknowledgement}

The author Mehmet KOCABIYIK would like to thank the Scientific and Technological Research Council of Turkey (TUBITAK 2211 programme) for the financial support.

\section{References}

[1] Cohen, F. (1987). Computer Viruses Theory and experiments, Computers and Security, vol.6, no.1, pp. 22-35.

[2] Szor, P. (2005). The Art of Computer Virus Research and Defense, Addison-Wesley

[3] Chen, L., Hattaf, K. and Sun, J. (2015). Optimal control of a delayed SLBS computer virus model. Physica A: Statistical Mechanics and its Applications, 427: 244-250.

[4] Yang, X. and Yang, L. X. (2012). Towards the epidemiological modeling of computer viruses. Discrete Dynamics in Nature and Society, (Article ID 259671).

[5] Yang, L. X. and Yang, X. (2014). A new epidemic model of computer viruses. Communications in Nonlinear Science and Numerical Simulation, 19(6): 1935-1944.

[6] Zhu, Q., Yang, X. and Ren, J. (2012). Modeling and analysis of the spread of computer virus. Communications in Nonlinear Science and Numerical Simulation, 17(12): 5117-5124.

[7] Lijuan, C., Khalid, H. and Jitao, S. (2015). Optimal control of a delayed SLBS computer virus model, Physica A: Statistical Mechanics and its Applications, 427: 244-250.

[8] Hoang, T. M., Dang, A. Q., and Dang, L. Q. (2018). Nonstandard finite difference schemes for solving a modified epidemiological model for computer viruses. Journal of Computer Science and Cybernetics, 34(2): 171-185.

[9] Barthelemy, M., Barrat, A., Pastor-Satorras, R. and Vespignani, A. (2004). Velocity and hierarchical spread of epidemic outbreaks in scale-free networks. Phys Re Lett; 92(7) (Article ID 178701).

[10] Karsai, M., Kivela, M., Pan, R.K., Kaski, K., Kertesz, J., Barabasi, A.L. and Saramaki, J. (2011). Small but slow world: How network topology and burstiness slow downspreading. Phys Rev E; 83(2) (Article ID 025102). 
[11] Billings, L., Spears, W.M. and Schwartz, I.B. (2002). A unied prediction of computer virus spread in connected networks. Phys Lett A; 297(3-4):261-6.

[12] Boguna ,M., Pastor-Satorras, R. and Vespignani, A. (2003). Absence of epidemic threshold in scale-free networks with degree correlations. Phys Rev Lett ;90(2)(Article ID 028701).

[13] Castellano, C. and Pastor-Satorras, R.. (2010). Thresholds for epidemic spreading in networks. Phys Rev Lett; 105(21) (Article ID 218701).

[14] Dezso, Z. and Barabasi, A.L. (2002). Halting viruses in scale-free networks. Phys Rev E; 65(5) (Article ID 055103).

[15] Fu, X., Small, M., Walker, D.M. and Zhang, H. (2008). Epidemic dynamics on scale- free networks with piecewise linear infectivity and immunization. Phys Rev E;77(3) (Article ID 036113).

[16] Lloyd, A.L. and May, R.M. (2001). How viruses spread among computers and people. Science; 292(5520):1316-7.

[17] Pastor-Satorras, R. and Vespignani, A. (2001). Epidemic spreading in scale-free networks. Phys Rev Lett ;86(14): 3200-3.

[18] Pastor-Satorras, R. and Vespignani, A. (2001). Epidemic dynamics and endemic states in complex networks. Phys Rev E ; 63(6) (Article ID 066117).

[19] Pastor-Satorras, R. and Vespignani, A. (2002). Immunization of complex networks. Phys Rev E; 65(3) (Article ID 036104).

[20] Wierman, J.C. and Marchette, D.J. (2004). Modeling computer virus prevalence with a susceptibleinfectedsusceptible model with reintroduction. Comput Stat Data Anal; 45(1):3-23.

[21] Chen, L.C. and Carley, K.M. (2004). The impact of countermeasure propagation on the prevalence of computer viruses. IEEE Trans Syst Man Cybern -Part B; 34(2): 823-33.

[22] Draief, M., Ganesh, A. and Massouilie, L. (2008). Thresholds for virus spread on networks. Ann Appl Probab; 18(2): 359-78.

[23] Griffin, C. and Brooks, R. (2006). A note on the spread of worms in scale-free networks. IEEE Trans Syst Man Cybern- Part B: Cybern; 36(1): 198-202.

[24] Moreno, Y., Pastor-Satorras, R. and Vespignani, A. (2002). Epidemic outbreaks in comple heterogeneus networks. Eur Phys J B; 26(4): 5219.

[25] Mickens,R.E. (1989). Exact solutions to a finite difference model of a nonlinear reaction advection equation: implications for numerical analysis. Numerical Methods for Partial Diferential Equations, vol. 5, no. 4, pp. 313-325.

[26] Mickens, R.E. (1990) Difference Equations Theory and Applications, Chapman Hall, Atlanta, Ga, USA.

[27] Mickens, R.E. (1993). Nonstandard Finite Difference Models of Differential Equations, World Scientic Publishing, Atlanta, Ga,USA.

[28] Mickens, R.E. (1999). Discretizations of nonlinear differential equations using explicit nonstandard methods. Journal of Computational and AppliedMathematics, 110(1): 181-185.

[29] Mickens, R.E. (1999). Applications of Nonstandard Finite Difference Schemes, World Scientic Publishing, Atlanta, Ga, USA. 
[30] Mickens, R.E. (2005). Advances in the Applications of Nonstandard Finite Difference Schemes,WileyInterscience, Singapore.

[31] Mickens, R.E. (2007). Calculation of denominator functions for nonstandard finite difference schemes for dierential equations satisfying a positivity condition, Numerical Methods for Partial Differential Equations, 23(3): 672-691, 2007.

[32] Mickens, R.E., Talitha M. W. (2013). NSFD discretizations of interacting population models satisfying conservation laws, Computers \& Mathematics with Applications.

[33] Zibaei, S. and Namjoo, M. (2014). A NSFD scheme for Lotka Volterra food web model. Iranian Journal of Science and Technology (Sciences), 38(4), 399-414.

[34] Cohn, A. (1922). Uber die Anzahl der Wurzeln einer algebraischen Gleichung in einem Kreise. Mathematische Zeitschrift, 14(1):110-148.

[35] Duffin, R. J. (1969). Algorithms for classical stability problems. Siam Review, 11(2):196-213.

[36] Elaydi, S.N. (1999). An Introduction to Difference Equations, Springer, New York, USA.

[37] Jury, E. (1964). I.Theory and Application of the z-Transform Method.

[38] Miller, J. J. (1971). On the location of zeros of certain classes of polynomials with applications to numerical analysis. IMA Journal of Applied Mathematics, 8(3), 397-406.

[39] Ongun, M. Y. and Turhan, I. (2013). A numerical comparison for a discrete HIV Infection of CD4+ T-Cell model derived from nonstandard numerical scheme. Journal of Applied Mathematics.

[40] Ongun, M.Y. and Özdoğan N. (2017). A nonstandard numerical scheme for a predator-prey model with Allee effect, J. Nonlinear Sci. Appl., 10:713-723.

[41] Schur, I. (1917). Uber Potenzreihen, die im Innern des Einheitskreises beschrankt sind. Journal für die reine und angewandte Mathematik, 147:205-232.

[42] Schur, I. (1918). Uber endliche Gruppen und Hermitesche Formen. Mathematische Zeitschrift, 1(2-3):184-207. 